DOI: 10.1002/adma.201502608

Article type: Communication

\title{
Structured Organic-inorganic Perovskite toward a Distributed Feedback Laser
}

Michael Saliba ${ }^{1 \dagger}$, Simon M. Wood ${ }^{2}$, Jay B. Patel ${ }^{1}$, Pabitra K. Nayak ${ }^{1}$, Jian Huang ${ }^{1}$, Jack A. Alexander-Webber ${ }^{1}$, Bernard Wenger ${ }^{1}$, Samuel D. Stranks ${ }^{1}$, Maximilian T. Hörantner ${ }^{1}$, Jacob Tse-Wei Wang ${ }^{1}$, Robin J. Nicholas ${ }^{1}$, Laura M. Herz ${ }^{1}$, Michael B. Johnston ${ }^{1}$, Stephen M. Morris $^{2}$, Henry J. Snaith ${ }^{1 *}$, and Moritz K. Riede ${ }^{1^{*}}$

${ }^{1}$ Clarendon Laboratory, University of Oxford, Parks Road, Oxford, OX1 3PU, U. K.

${ }^{2}$ Department of Engineering Science, University of Oxford, Parks Road, Oxford, OX1 3PJ, U.K.

\section{Corresponding authors}

* henry.snaith@physics.ox.ac.uk, moritz.riede@physics.ox.ac.uk

\section{Present Address}

${ }^{\dagger}$ Laboratory of Photonics and Interfaces, École polytechnique fédérale de Lausanne, Station 6, 1015 Lausanne, Switzerland

Keywords: perovskite, distributed feedback laser, nanoimprinting, evaporation

Organic-inorganic halide perovskite materials, $\mathrm{APbX}_{3}(\mathrm{~A}=$ methylammonium (MA),

formamidinium; $\mathrm{X}=\mathrm{Br}, \mathrm{I}$ ), have rapidly emerged as exciting light-harvesting materials with dramatically improved solar power conversion efficiencies from $3.8 \%$ in $2009^{[1]}$ to $20.1 \%$ in 2014. ${ }^{[2]}$ These materials exhibit remarkably high absorption over the visible spectrum, ${ }^{[3]} \mathrm{a}$ direct bandgap, ${ }^{[4]}$ and charge carrier diffusion lengths in the micrometer-range ${ }^{[5,6]}$ implying a sharp optical band edge with low trap densities. ${ }^{[7]}$ Perovskite thin films can be deposited by a variety of techniques including: one step deposition from a metal-halide precursor solution, ${ }^{[3,8,}$ ${ }^{9]}$ two step processing where a metal-halide layer is subsequently converted to perovskite by exposure to an organic component, ${ }^{[10-12]}$ and/or simultaneous co-evaporation of the respective perovskite precursor components. ${ }^{[13,14]}$ Importantly, by varying the cation, metal or anion in the perovskite, the electronic band-gap may be tuned continuously between 1.1 and $3.1 \mathrm{eV} .^{[15-}$ ${ }^{17]}$ It is this combination of facile manufacturability, optical tuneability, and material 
properties that has heralded a new branch of research into light-emission applications using perovskites.

Previous works on related perovskite structures have demonstrated biexciton lasing at $40 \mathrm{~K}^{[18]}$ and electroluminescence at liquid nitrogen temperatures, ${ }^{[19,20]}$ leading to early perovskite light-emitting diodes (LEDs). ${ }^{\left[20,{ }^{21]}\right.}$ More recently, perovskites similar to $\mathrm{MAPbI}_{3}$, which is used widely for solar cells, resulted in room temperature LEDs, ${ }^{[22]}$ as well as amplified spontaneous emission $^{[23]}$ and lasing when the perovskite is sandwiched between a gold mirror and a dielectric stack. ${ }^{[24]}$ Following this, amplified emission has been demonstrated in microcrystal networks, ${ }^{[25]}$ spherical resonators, ${ }^{[26]}$ polygonal nanoplatelets, ${ }^{[27]}$, cholesteric liquid crystals ${ }^{[28]}$ and nanowires. ${ }^{[29]}$ However, these architectures frequently require laborious techniques for integrating mirrors to form the optical cavity, and often exhibit poor optical tuning behaviour as well as no single mode emission, potentially barring the application of these cavities as routes to low-cost commercial solid state laser diodes. Thus, achieving single mode operation (with a single frequency and narrow emission) using a device architecture that is compatible with scalable low-cost manufacturing still remains a challenge.

One design that can meet industrial demands is the distributed feedback (DFB) cavity. In this structure, optical feedback is provided by Bragg scattering from an interference grating built either directly into the active medium or within the vicinity of the whole resonator by periodically alternating the refractive index. Pioneered by Kogelnik et al. in $1971,{ }^{[30]}$ DFB lasers have become the standard for many industrial applications due to their inexpensive manufacturability, low thresholds, high quality factor (QF), mirror-free design, narrow linewidth and most importantly tuneable single mode output over a wide spectral range. ${ }^{[31]}$ Successful DFB systems have been realized using a myriad of active gain media including semiconducting polymers, ${ }^{[32]}$ dyes, ${ }^{[30]}$ organic,${ }^{[33]}$ and inorganic materials. ${ }^{[34]}$ In Figure 1a, we show a schematic diagram of a corrugated DFB structure consisting of two media with refractive indices $n_{1}$ and $n_{2}$. In this geometry, the Bragg conditions can be 


\section{WILEY-VCH}

expressed as ${ }^{[30]} 2 n_{\text {eff }} \Lambda=m \lambda_{\text {Bragg }}$, where $n_{\text {eff }}$ is the effective refractive index of the combined structured polymer-perovskite-air medium, $\Lambda$ is the periodicity of the corrugated structure, $m$ is the diffraction order, and $\lambda_{\text {Bragg }}$ is the Bragg wavelength. First order Bragg scattering $(m=1)$ results in an edge-emitting mode, while second order Bragg scattering $(m=2)$ results in a surface-emitting mode. From this, it is clear that the DFB design only works for a precisely patterned active medium.

However, structuring perovskite thin films with this degree of precision has not been demonstrated yet. Therefore, it is crucial to develop a practical route to implement a perovskite-based DFB structure which is critical for assessing the potential industrial impact of perovskite lasers. One very common approach for fabricating organic DFB lasers is the direct nanoimprinting of the active material. ${ }^{[33,35,36]}$ However, it is important to note the distinction between organics (soft materials) and perovskites (hard materials). It is not directly obvious that a technique used to enable lasing in organics would be translatable to perovskites. Indeed, it still remains an open challenge to demonstrate direct nanoimprinting of perovskite films.

In this work, we realise for the first time a perovskite DFB cavity by nanoimprinting a corrugated structure onto a polymer template, followed by the subsequent evaporation of a conformal perovskite layer. More specifically, we manufacture corrugated perovskite films with periodicities from 370-440 $\mathrm{nm}$ by nanoimprinting a UV-curable polymer resist, coated on a glass substrate. Then, the textured polymer films get coated by a film of $\sim 120$-nm thick perovskite, which is deposited by a dual source co-evaporator of methylammonium iodide and lead chloride according to the procedure developed by Liu et al. ${ }^{[13]}$ The photoluminescence quantum efficiency of these films was determined to be $\sim 16 \%$. We patterned areas of $0.27 \mathrm{~cm}^{2}$ and up to $1.6 \mathrm{~cm}^{2}$ which are both sufficiently large to observe interference effects by eye from the resulting corrugated structures (see Figure S1 in the Supporting Information). Full fabrication details can be found in the Materials \& Methods section. 


\section{WILEY-VCH}

In Figure 1b-d, we present atomic force microscopy (AFM) images demonstrating the successful pattern transfer onto the polymer resist via nanoimprinting for a periodicity of $410 \mathrm{~nm}$ which was patterned over an area of $0.27 \mathrm{~cm}^{2}$.

We show the full glass/corrugated polymer/evaporated perovskite DFB stack in the AFM image in Figure 1e and the corresponding height profile in Figure 1f and have included additional SEM images in the Supporting Information (Figure S2) showing clearly that the perovskite layer is corrugated. Similar results are obtained for the other periodicities as well. From this, we conclude that the evaporated perovskite forms a conformal coating on the underlying corrugated polymer resist.

Our approach provides for the first time a general strategy for introducing $2 \mathrm{D}$ in-plane optical structuring of perovskite films, which could be expanded to any feasible $2 \mathrm{D}$ pattern. The DFB cavity is a first concrete application proving the concept of our approach, which is also specifically a critical step towards injection laser diodes. Hence, this generic structuring principle opens the prospects of the perovskite material for much improved optical control not only in LEDs and solar cells, but also toward applications as optical devices, through in-plane wave-guiding or plasmonics. ${ }^{[37-40]}$

Our experiments focus on a surface-emitting DFB cavity, i.e. 2nd order Bragg scattering. We screened through eight different grating periodicities from 370 to $440 \mathrm{~nm}$ in steps of $10 \mathrm{~nm}$ including an unpatterned reference area (see Figure S1 for images of the master stamps used). In Figure 2a-c, we present the emission spectra of the full glass/corrugated polymer/perovskite stack when optically pumped from the glass side of the substrate at $\lambda=532 \mathrm{~nm}$ with 1 -ns pulses, a repetition rate of $1 \mathrm{kHz}$ and excitation fluences (or pump powers) varying from 0.04 to $4.35 \mu \mathrm{J} / \mathrm{cm}^{2} /$ pulse for three different grating periodicities 


\section{WILEY-VCH}

of $\Lambda=400 \mathrm{~nm}, 410 \mathrm{~nm}$, and $420 \mathrm{~nm}$ which were all patterned on the same perovskite film to ensure comparability.

A clear trend for all periodicities is the emergence of a peak at $760 \mathrm{~nm}$, which is at a slightly shorter wavelength than the steady-state photoluminescence peak, and continues to increase with higher excitation intensities. In addition, an amplified emission peak of much narrower linewidth occurs at a higher wavelength at $779 \mathrm{~nm}$ for $\Lambda=400 \mathrm{~nm}, 784 \mathrm{~nm}(\Lambda=400 \mathrm{~nm})$, and $793 \mathrm{~nm}(\Lambda=420 \mathrm{~nm})$.

As an example, for $\Lambda=400 \mathrm{~nm}$, the amplified emission peak increases rapidly with increasing excitation fluences accompanied by a narrow full width at half maximum (FWHM) of $2.2 \mathrm{~nm}$ for the highest excitation fluence at $4.35 \mu \mathrm{J} / \mathrm{cm}^{2} /$ pulse. This trend can also be seen for grating periodicities of $\Lambda=410$ and $420 \mathrm{~nm}$ where the narrow peak increases rapidly in intensity with FWHMs of $2.1 \mathrm{~nm}$, and $1.4 \mathrm{~nm}$, respectively.

In Figure 2d-f, we show the input-output characteristics by extracting the narrow peak intensity from the emission spectra as a function of the excitation fluence. At first, we note a linear increase in the intensity (due to spontaneous emission) that is then superseded by a rapid increase at the respective fluence thresholds of $\sim 0.32(\Lambda=400 \mathrm{~nm}), \sim 0.54(\Lambda=410 \mathrm{~nm})$, and $\sim 2.11(\Lambda=420 \mathrm{~nm}) \mu \mathrm{J} / \mathrm{cm}^{2} /$ pulse, which is significantly lower than most values of $\sim 10$ $20 \mu \mathrm{J} / \mathrm{cm}^{2} /$ pulse reported in literature for lasing structures with similar perovskites. ${ }^{[24,23]}$ We could reproducibly observe this effect for various devices imprinted with a single stamp over an area of $1.6 \mathrm{~cm}^{2}$ and a periodicity of $\Lambda \sim 416 \mathrm{~nm}$. We present these results in Figure S3 of the Supporting Information where amplified emission can be seen at $796 \mathrm{~nm}$ (FWHM of $1.4 \mathrm{~nm}$ ) with a threshold of $\sim 1 \mu \mathrm{J} / \mathrm{cm}^{2} /$ pulse. It is noteworthy that our results were realised on simple glass/polymer/perovskite stacks without any additional layers - highlighting the simplicity of manufacture.

Furthermore, the amplified emission peak is found to red-shift as the grating period is increased from 400 to $420 \mathrm{~nm}$. To elucidate this point further, we present in Figure 3 the 


\section{WILEY-VCH}

magnified emission spectra of these three periodicities at the highest fluence $\left(4.35 \mu \mathrm{J} / \mathrm{cm}^{2} /\right.$ pulse) supplemented by another 2 periodicities (380 and $390 \mathrm{~nm}$ ) which were measured at a later time on a separate sample. We also include a control device (dashed line) from an unpatterned part of the film along with an inset of the peak wavelength as a function of the periodicity. The QF of the DFB cavity could not be determined accurately due to the resolution limit of the spectrometer. However, in comparison with similar organic DFB structure we estimate that it should exceed the apparent value (QF 400).

The inset of Figure 3 displays peak wavelength as a function of the periodicity showing a redshift with increasing periodicity. Hence, our findings for the amplified emission peak are a significant narrowing of the linewidth with increased excitation fluences, which cannot be seen for a control device, a clear threshold, and a controlled red-shift of the wavelength as the grating periodicity of the DFB cavity is increased. This constitutes at least amplified spontaneous emission (ASE) and shows that our structuring approach resulted successfully in the first perovskite DFB cavity. We note that lasing has already been proven in perovskites ${ }^{[24]}$, and provided all other characteristics are consistent with lasing, we may already observe lasing action. However, due to the challenges to clearly classify that the observed emission is lasing, which would require highly specialised photon statistics measurements, we refrain from calling our observations lasing.

Furthermore, while we observe a red-shift of the wavelength with increasing periodicity, we also note that the shift does not follow the Bragg scattering law with a constant refractive index. Assuming an effective refractive index of $n_{\text {eff }}=1.9$ or 2.0 (motivated by simulations in Table S1 where we found $n_{\text {eff }}=1.916$.) and using the 2nd order Bragg equation $\lambda_{\text {Bragg }}=n_{\text {eff }} \times \Lambda$, we would expect the modes to be separated by at least $19 \mathrm{~nm}$ (see Figure S4a) instead of the observed $\sim 5 \mathrm{~nm}$. This discrepancy can be explained by the dispersion of the refractive indices. In Figure S4b, we calculate what the effective refractive index should be in order to match the observed modes. From this, $n_{\text {eff }}$ drops from $\sim 2.0$ (for $\Lambda=380 \mathrm{~nm}$ and 
$\lambda_{\text {measured }}=770 \mathrm{~nm}$ ) to $n_{\text {eff }} \sim 1.88$ (for $\Lambda=420 \mathrm{~nm}$ and $\lambda_{\text {measured }}=793 \mathrm{~nm}$ ) showing the potential impact of even small changes in refractive index. This behaviour is supported by refractive index measurements of similar perovskite materials by Ball ${ }^{[41]}$ Loper $^{[42]}$ and Lin $e t$ $a l .{ }^{[43]}$ who all report a decline in refractive index from $770-800 \mathrm{~nm}$ with a relative difference of up to $\Delta n \sim 0.1$. This is in agreement with our calculations.

Furthermore, using the Bragg scattering law is only a first order approximation and simulations are required to get a more realistic picture. To this end, we calculate the fundamental DFB waveguide modes for a periodicity of $400 \mathrm{~nm}$ by solving numerically the phase-matching conditions ${ }^{[44]}$ for a three-layer waveguide consisting of a polymer substrate, the perovskite film and air as the cover medium. To demonstrate the impact of the different refractive indices of perovskite as reported in literature, we use one of the smallest reported values in the $770-800 \mathrm{~nm}$ range: $n_{800 \mathrm{~nm}, \text { Ball }}=2.507$ by Ball et al. ${ }^{[41]}$, and one of the largest reported values: $n_{770 \mathrm{~nm}, \mathrm{Lin}}=2.853$ by Lin et al. ${ }^{[43]}$ as well as the full dispersion relation by Ball et al. ${ }^{[41]}$. This permits for a realistic range of values with which we can cross check our experimental results. In Figure S5, we present the calculated fundamental mode for a DFB grating with a periodicity of $400 \mathrm{~nm}$ as a function of perovskite film thickness. For $120 \mathrm{~nm}$, we expect the DFB mode from $770-800 \mathrm{~nm}$, depending on the refractive index, highlighting the importance of accurate dielectric constant data. The mode is in good agreement with our experimental results.

In summary, we have presented a new strategy for structuring organic-inorganic perovskite materials for optoelectronic applications. By conformally evaporating perovskite onto a nanoimprinted polymer resist, we were able to create a working perovskite DFB cavity and to tune the emission between 770 and $793 \mathrm{~nm}$ simply by varying the grating periodicity. Our experimental findings are further supported by optical simulations. This is the first time a general structuring approach for perovskite thin films is presented which can be expanded to 


\section{WILEY-VCH}

any feasible 2D pattern. DFB perovskite cavities have huge potential as inexpensive, mirror free, widely-tuneable, single mode lasers that are easy to manufacture on a large scale. The DFB structure is highly versatile and can be optimised - for example towards lower thresholds or different output energies. Broad tuneability may be achieved by utilising the various closely related organic-inorganic perovskites and tailoring the cavity to the gain maximum of the unpatterned film. With this approach, continuous tuning of the emission from $1.1-3.1 \mathrm{eV}$ should be feasible which has relevance for telecommunication, medicine and many other applications. Furthermore, our work provides a crucial step to the exciting prospect of an all-electrically pumped perovskite laser- a device that will have far reaching consequences and a multitude of commercial applications including sensors, displays, guidance systems, and lab-on-chip technology.

\section{Experimental Section}

Lead chloride (CAS No. 6080-56-4) was purchased from SigmaAldrich.

Methylammonium iodide $\left(\mathrm{CH}_{3} \mathrm{NH}_{3} \mathrm{I}\right)$ was prepared by reacting methylamine (33 wt $\%$ in ethanol, Sigma-Aldrich) with hydroiodic acid (HI) (57 wt\% in water, Sigma-Aldrich) at room temperature. $\mathrm{HI}$ was added drop-wise while stirring. Upon drying at $100{ }^{\circ} \mathrm{C}$, a white powder was formed, which was dried overnight in a vacuum oven and purified with ethanol before use.

\section{Electron-beam (e-beam) lithography}

To achieve optical feedback, the Bragg wavelength needs to match the photoluminescence (PL) spectrum of a $\mathrm{CH}_{3} \mathrm{NH}_{3} \mathrm{PbI}_{3}$ perovskite film, which has an emission peak at $780 \mathrm{~nm}$ with a spectral range from $740-820 \mathrm{~nm} \cdot{ }^{[13,5]}$ Furthermore, to ensure maximum efficiency, the grating period needs to match the Bragg wavelength with the gain maximum of the material. Therefore, we used as a first order approximation, $m=2$ (surface-emitting), assumed 


\section{WILEY-VCH}

$n_{\text {eff }}=1.85^{[43]}$ (effective refractive index of the combined polymer-perovskite-air medium), and a perovskite PL wavelength of $\sim 780 \mathrm{~nm}$, resulting in a periodicity of $\Lambda \sim 420 \mathrm{~nm}$.

Thus, a large area $(2 \mathrm{~cm} \times 2 \mathrm{~cm})$ nanoimprint lithography stamp was fabricated on a $200 \mathrm{~nm}$ thick $\mathrm{Si} / \mathrm{Cr}$ substrate. The stamp was designed to contain multiple gratings with $100 \mathrm{~nm}$ deep $\mathrm{Cr}$ ridges and periodicities ranging in $10 \mathrm{~nm}$ steps from $370-440 \mathrm{~nm}$. This is referred to as a "multistamp" here. The periodicities from $370-440 \mathrm{~nm}$ covered an area or "pixel" of $0.6 \mathrm{~cm} \times 0.45 \mathrm{~cm}$. An additional pixel was only partially covered to allow for a large unpatterned area for control experiments.

A bilayer polymethylmethacrylate (PMMA) resist, with molecular weights of $495 \mathrm{k}(6 \%$ in anisole) and 950k (3\% in anisole) respectively, was spun onto the $\mathrm{Si} / \mathrm{Cr}$ substrate at $6000 \mathrm{rpm}$ and pre-baked at $175^{\circ} \mathrm{C}$ for $5 \mathrm{~min}$, and was then patterned according to the above design using a JEOL JBC-5500ZC electron beam lithography system.

Subsequently $100 \mathrm{~nm}$ of Cr was thermally evaporated onto the patterned resist and lift-off was performed in hot N-Methyl-2-pyrrolidone (NMP) with mild sonication. Scanning electron microscopy confirmed the uniformity of the features across large areas of the sample which was then used as a hard stamp for creating further soft nanoimprint lithography masks.

\section{Nanoimprinting Lithography (NIL)}

For the nanoimprinting, two different hard stamps were employed - the multistamp described above and a Thorlabs diffraction grating with a periodicity of $\sim 416 \mathrm{~nm}$ (2400 grooves $/ \mathrm{mm}$, $1.27 \mathrm{~cm} \times 1.27 \mathrm{~cm}$, part number: GH13-24U).

A soft stamp was fabricated from these hard stamps using Obducat's Eitre nanoimprint lithography (NIL) system. The hard stamp was pressed against a flexible intermediate polymer stamp (IPS, Obducat) while holding the temperature at $155^{\circ} \mathrm{C}$ and the pressure at 40 bar. 


\section{WILEY-VCH}

Next, a microscope slide was cleaned with acetone, ethanol and exposure to an oxygen plasma treatment for $10 \mathrm{~min}$. Then, Obducat's STU (Simultaneous Thermal and UV) resist was spin-coated at $2000 \mathrm{rpm}$ for $120 \mathrm{~s}$ resulting in $\sim 100 \mathrm{~nm}$ thick films.

The soft stamp was used to transfer the patterns onto the microscope slide by a simultaneous thermal and UV imprint process. Throughout the $3.5 \mathrm{~min}$ long procedure the temperature was $70{ }^{\circ} \mathrm{C}$ and the pressure 30 bar. After $30 \mathrm{~s}$, the polymer resist was exposed to UV light for $60 \mathrm{~s}$. Thereafter, the soft stamp was lifted off the microscope slide.

The successful pattern transfer was confirmed by SEM images and the interference pattern from the corrugated structure that could be seen by eye.

\section{Evaporation}

Thin perovskite films were deposited by dual-source evaporation, as described in ref ${ }^{[13]}$. Lead chloride $\left(\mathrm{PbCl}_{2}\right)$ and methylammonium iodide $\left(\mathrm{CH}_{3} \mathrm{NH}_{3} \mathrm{I}\right)$ were deposited simultaneously onto nanoimprinted substrates (see above) under high vacuum. $500 \mathrm{mg}$ of $\mathrm{CH}_{3} \mathrm{NH}_{3} \mathrm{I}$ and $100 \mathrm{mg}$ of $\mathrm{PbCl}_{2}$ were loaded into separate crucibles and the substrate was mounted on a holder above the sources. In the next step, volatile impurities were removed by heating the two crucibles above the desired deposition temperatures under high vacuum $\left(10^{-5} \mathrm{mbar}\right)$ for $5 \mathrm{~min}$. To prepare representative perovskite films, key deposition parameters such as the deposition rates and duration for the two sources were set in guidance with the previously optimized for best performance of the material in solar cells ${ }^{[13]}$. This includes using a $\mathrm{CH}_{3} \mathrm{NH}_{3} \mathrm{I}: \mathrm{PbCl}_{2}$ molar ratio of $4: 1$ and heating the $\mathrm{CH}_{3} \mathrm{NH}_{3} \mathrm{I}$ to around $115^{\circ} \mathrm{C}$ and $\mathrm{PbCl}_{2}$ to $330^{\circ} \mathrm{C}$. The substrate holder was kept at $21^{\circ} \mathrm{C}$ and was rotated to ensure uniform coating while the film was deposited on the substrate. The deposition rate and the thickness of the films were monitored using a quartz crystal microbalance. Subsequently, the perovskite films were fully crystalized in a nitrogen-filled glove box by annealing at $100{ }^{\circ} \mathrm{C}$ for 30-45 min depending on the thickness of the film. For the multistamp patterns, $\sim 120 \mathrm{~nm}$ 


\section{WILEY-VCH}

thick perovskite films were used and $\sim 80 \mathrm{~nm}$ for the single stamp pattern (Thorlab diffraction grating).

\section{Optical characterisation}

The emission properties of the devices were tested by pumping with a Nd:YAG laser (6FTSS355-Q4-S) operating at $532 \mathrm{~nm}$ with 1-ns pulses at repetition rate of up to $1 \mathrm{kHz}$. The laser was focused onto the device from the glass side using a focusing lens to a spot size of $100 \mu \mathrm{m}$. The device output was collected using an objective and focussed with an achromatic collimating lens into a $600 \mu \mathrm{m}$ diameter fibre coupled to a spectrometer (Ocean Optics USB2000+, $1.7 \mathrm{~nm}$ resolution).

Scanning electron microscopy (SEM) images were acquired on Pd-coated films using a Hitachi S-4300 SEM.

Atomic force microscopy (AFM) images were taken using a ThermoMicroscopes AutoProbe M5 housed in an acoustic isolation enclosure in non-contact mode, with a scan rate of $0.2 \mathrm{~Hz}$ and $256 \times 256$ pixels. Aluminum coated antimony doped Si tips (Bruker) were used, with typical tip radii of $8 \mathrm{~nm}$, resonant frequencies of $75 \mathrm{kHz}$, and stiffness of $3 \mathrm{~N} / \mathrm{m}$.

Photoluminescence quantum efficiency (PLQE) values were determined using a $532 \mathrm{~nm} \mathrm{CW}$ laser excitation source (Suwtech LDC-800) to illuminate a sample in an integrating sphere (Oriel Instruments 70682NS), and the laser scatter and PL collected using a fiber-coupled detector (Ocean Optics USB 2000+). The spectral response of the fiber-coupled detector setup was calibrated using a spectral irradiance standard (Oriel Instruments 63358). Excitation intensities were adjusted using optical density filters and the highest intensities were obtained using a $25 \mathrm{~cm}$ focusing lens to reduce the spot size. PLQE calculations were carried out using established techniques. ${ }^{[45]}$

\section{Simulations}

The effective indices of the waveguide modes are estimated by solving numerically the phase- 


\section{WILEY-VCH}

matching conditions ${ }^{[44]}$ for a three-layer waveguide consisting of a polymer substrate

$\left(n_{\text {polymer }}=1.52\right)$, the perovskite film $\left(n_{\text {perovskite }}=2.56\right)$ and air as the cover medium $\left(n_{\text {air }}=1.00\right)$.

The refractive indices of the different materials are fixed at $780 \mathrm{~nm}$ and later the dispersion

relation for perovskite is taken into account.

\section{Supporting Information}

Supporting Information is available from the Wiley Online Library or from the author.

\section{Acknowledgements}

MS, HJS, and MKR conceived and designed the experiments for this work. SMW (supervised by SMM) conducted all optical characterisation. MS assembled the overall device stack including the nanoimprinting processing. JH, JAAW (supervised by RJN) designed and conducted e-beam lithography as well as AFM measurements. JBP (supervised by MBJ and LMH) optimised and evaporated the perovskite layer. BW conducted the simulations. MTH, PKN, SDS (supervised by HJS), and JTWW (supervised by RJN) conducted SEM imaging, as well as fabricated and analysed additional experiments for optimization.

MS wrote the first draft of the paper. All authors contributed to the writing of the paper.

We thank the anonymous reviewers for a helpful discussion which has improved this work. We thank Prof. Robert Taylor and Dr. Benjamin Reid for helpful discussions. We thank Dr. Jin Zhan for preparing the methylammonium iodide powder and Sven M. Hein for assisting with the TOC figure.

The research leading to these results has received funding from the European Union Seventh Framework Programme [FP7/2007-2013] under grant agreement 604032 of the MESO project. SMM gratefully acknowledges The Royal Society for Financial Support. We acknowledge ERSRC funding.

Received: ((will be filled in by the editorial staff))

Revised: ((will be filled in by the editorial staff)) Published online: ((will be filled in by the editorial staff))

[1] A. Kojima, K. Teshima, Y. Shirai, T. Miyasaka, J Am Chem Soc 2009, 131, 6050.

[2] W. S. Yang, J. H. Noh, N. J. Jeon, Y. C. Kim, S. Ryu, J. Seo, S. I. Seok, Science 2015.

[3] M. M. Lee, J. Teuscher, T. Miyasaka, T. N. Murakami, H. J. Snaith, Science 2012, $338,643$.

[4] J. Even, L. Pedesseau, C. Katan, J Phys Chem C 2014, 118, 11566.

[5] S. D. Stranks, G. E. Eperon, G. Grancini, C. Menelaou, M. J. P. Alcocer, T. Leijtens, L. M. Herz, A. Petrozza, H. J. Snaith, Science 2013, 342, 341.

[6] G. Xing, N. Mathews, S. Sun, S. S. Lim, Y. M. Lam, M. Gratzel, S. Mhaisalkar, T. C. Sum, Science 2013, 342, 344.

[7] H. Oga, A. Saeki, Y. Ogomi, S. Hayase, S. Seki, Journal of the American Chemical Society 2014, 136, 13818. 


\section{WILEY-VCH}

[8] M. Saliba, K. W. Tan, H. Sai, D. T. Moore, T. Scott, W. Zhang, L. A. Estroff, U. Wiesner, H. J. Snaith, J Phys Chem C 2014, 118, 17171.

[9] N. J. Jeon, J. H. Noh, W. S. Yang, Y. C. Kim, S. Ryu, J. Seo, S. I. Seok, Nature 2015, $517,476$.

[10] J. Burschka, N. Pellet, S. J. Moon, R. Humphry-Baker, P. Gao, M. K. Nazeeruddin, M. Gratzel, Nature 2013, 499, 316.

[11] Q. Chen, H. P. Zhou, Z. R. Hong, S. Luo, H. S. Duan, H. H. Wang, Y. S. Liu, G. Li, Y. Yang, Journal of the American Chemical Society 2014, 136, 622.

[12] Z. G. Xiao, C. Bi, Y. C. Shao, Q. F. Dong, Q. Wang, Y. B. Yuan, C. G. Wang, Y. L. Gao, J. S. Huang, Energy \& Environmental Science 2014, 7, 2619.

[13] M. Z. Liu, M. B. Johnston, H. J. Snaith, Nature 2013, 501, 395.

[14] O. Malinkiewicz, A. Yella, Y. H. Lee, G. M. Espallargas, M. Graetzel, M. K.

Nazeeruddin, H. J. Bolink, Nat Photonics 2014, 8, 128.

[15] J. H. Noh, S. H. Im, J. H. Heo, T. N. Mandal, S. I. Seok, Nano Lett 2013, 13, 1764.

[16] F. Hao, C. C. Stoumpos, R. P. H. Chang, M. G. Kanatzidis, Journal of the American Chemical Society 2014, 136, 8094.

[17] Y. Ogomi, A. Morita, S. Tsukamoto, T. Saitho, N. Fujikawa, Q. Shen, T. Toyoda, K. Yoshino, S. S. Pandey, T. L. Ma, S. Hayase, J Phys Chem Lett 2014, 5, 1004.

[18] T. Kondo, T. Azuma, T. Yuasa, R. Ito, Solid State Commun 1998, 105, 253.

[19] X. Hong, T. Ishihara, A. V. Nurmikko, Solid State Commun 1992, 84, 657.

[20] M. Era, S. Morimoto, T. Tsutsui, S. Saito, Appl Phys Lett 1994, 65, 676.

[21] T. Gebauer, G. Schmid, Zeitschrift Fur Anorganische Und Allgemeine Chemie 1999, $625,1124$.

[22] Z. K. Tan, R. S. Moghaddam, M. L. Lai, P. Docampo, R. Higler, F. Deschler, M. Price, A. Sadhanala, L. M. Pazos, D. Credgington, F. Hanusch, T. Bein, H. J. Snaith, R. H. Friend, Nat Nanotechnol 2014, 9, 687.

[23] G. Xing, N. Mathews, S. S. Lim, N. Yantara, X. Liu, D. Sabba, M. Gratzel, S.

Mhaisalkar, T. C. Sum, Nat Mater 2014, 13, 476.

[24] F. Deschler, M. Price, S. Pathak, L. E. Klintberg, D. D. Jarausch, R. Higler, S. Huttner, T. Leijtens, S. D. Stranks, H. J. Snaith, M. Atature, R. T. Phillips, R. H. Friend, J Phys Chem Lett 2014, 5, 1421.

[25] R. Dhanker, A. N. Brigeman, A. V. Larsen, R. J. Stewart, J. B. Asbury, N. C. Giebink, Appl Phys Lett 2014, 105.

[26] B. R. Sutherland, S. Hoogland, M. M. Adachi, C. T. Wong, E. H. Sargent, Acs Nano 2014, 8, 10947.

[27] Q. Zhang, S. T. Ha, X. Liu, T. C. Sum, Q. Xiong, Nano Lett 2014, 14, 5995.

[28] S. D. Stranks, S. M. Wood, K. Wojciechowski, F. Deschler, M. Saliba, H. Khandelwal, J. B. Patel, S. J. Elston, L. M. Herz, M. B. Johnston, A. P. H. J. Schenning, M. G. Debije, M. K. Riede, S. M. Morris, H. J. Snaith, Nano Lett 2015, 15, 4935.

[29] H. Zhu, Y. Fu, F. Meng, X. Wu, Z. Gong, Q. Ding, M. V. Gustafsson, M. T. Trinh, S. Jin, X. Y. Zhu, Nat Mater 2015, advance online publication.

[30] H. Kogelnik, C. V. Shank, Appl Phys Lett 1971, 18, 152.

[31] J. E. Carroll, J. Whiteaway, D. Plumb, Distributed feedback semiconductor lasers, Vol. 10, IET, London 1998.

[32] N. Tessler, G. J. Denton, R. H. Friend, Nature 1996, 382, 695.

[33] M. Berggren, A. Dodabalapur, R. E. Slusher, A. Timko, O. Nalamasu, Appl Phys Lett 1998, 72, 410.

[34] T. N. Smirnova, O. V. Sakhno, P. V. Yezhov, L. M. Kokhtych, L. M. Goldenberg, J. Stumpe, Nanotechnology 2009, 20, 245707.

[35] D. Pisignano, L. Persano, P. Visconti, R. Cingolani, G. Gigli, G. Barbarella, L. Favaretto, Appl Phys Lett 2003, 83, 2545. 


\section{WILEY-VCH}

[36] E. B. Namdas, M. Tong, P. Ledochowitsch, S. R. Mednick, J. D. Yuen, D. Moses, A. J. Heeger, Adv Mater 2009, 21, 799.

[37] M. Hentschel, M. Saliba, R. Vogelgesang, H. Giessen, A. P. Alivisatos, N. Liu, Nano Lett 2010, 10, 2721.

[38] M. Mader, T. Hoche, J. W. Gerlach, S. Perlt, J. Dorfmuller, M. Saliba, R. Vogelgesang, K. Kern, B. Rauschenbach, Nano Lett 2010, 10, 47.

[39] W. Zhang, M. Saliba, S. D. Stranks, Y. Sun, X. Shi, U. Wiesner, H. J. Snaith, Nano Lett 2013, 13, 4505.

[40] M. Saliba, W. Zhang, V. M. Burlakov, S. D. Stranks, Y. Sun, J. M. Ball, M. B. Johnston, A. Goriely, U. Wiesner, H. J. Snaith, Adv Funct Mater 2015, 25, 5038.

[41] J. M. Ball, S. D. Stranks, M. T. Hoerantner, S. Huettner, W. Zhang, E. J. W. Crossland, I. Ramirez, M. Riede, M. B. Johnston, R. H. Friend, H. J. Snaith, Energy \& Environmental Science 2015, 8, 602.

[42] P. Loper, M. Stuckelberger, B. Niesen, J. Werner, M. Filipic, S. J. Moon, J. H. Yum, M. Topic, S. De Wolf, C. Ballif, J Phys Chem Lett 2015, 6, 66.

[43] Q. Q. Lin, A. Armin, R. C. R. Nagiri, P. L. Burn, P. Meredith, Nat Photonics 2015, 9, 106.

[44] K. Okamoto, Fundamentals of optical waveguides, Academic Press, Elsevier, Burlington, USA 2010.

[45] J. C. deMello, H. F. Wittmann, R. H. Friend, Adv Mater 1997, 9, 230. 


\section{WILEY-VCH}
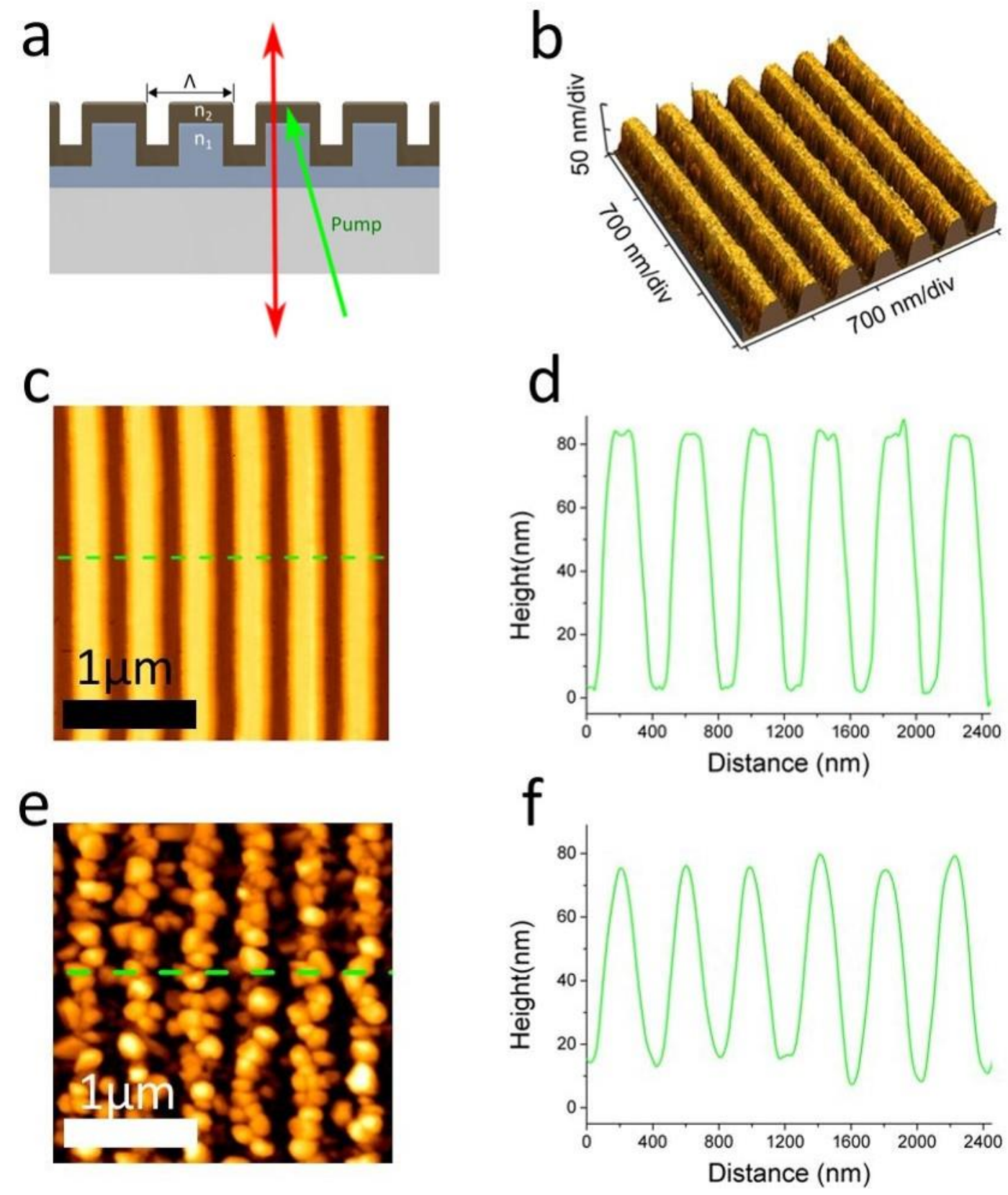

Figure 1. (a) Schematic diagram of a generic distributed feedback (DFB) cavity with glass/patterned polymer resist/perovskite structure. The periodicity is $\Lambda$, the refractive index for the polymer is $\mathrm{n}_{1}$ and $\mathrm{n}_{2}$ for the perovskite layer. Upon optical pumping, the DFB structure responds with surface emission in the case of second order Bragg scattering.

Atomic force microscopy (AFM) images of the patterned polymer resist in 3D (b) and 2D (c).

(d) Height profile in the direction of the green dashed line (averaged over $4 \mu \mathrm{m}$ ) in (c) confirming the periodicity of $410 \mathrm{~nm}$

(e) AFM image (top view) after the perovskite is evaporated on the polymer resist. 


\section{WILEY-VCH}

(f) Height profile of the patterned perovskite obtained in direction of the green dashed line

(averaged over $4 \mu \mathrm{m}$ ) in (e) confirming the periodic and conformal perovskite coating.
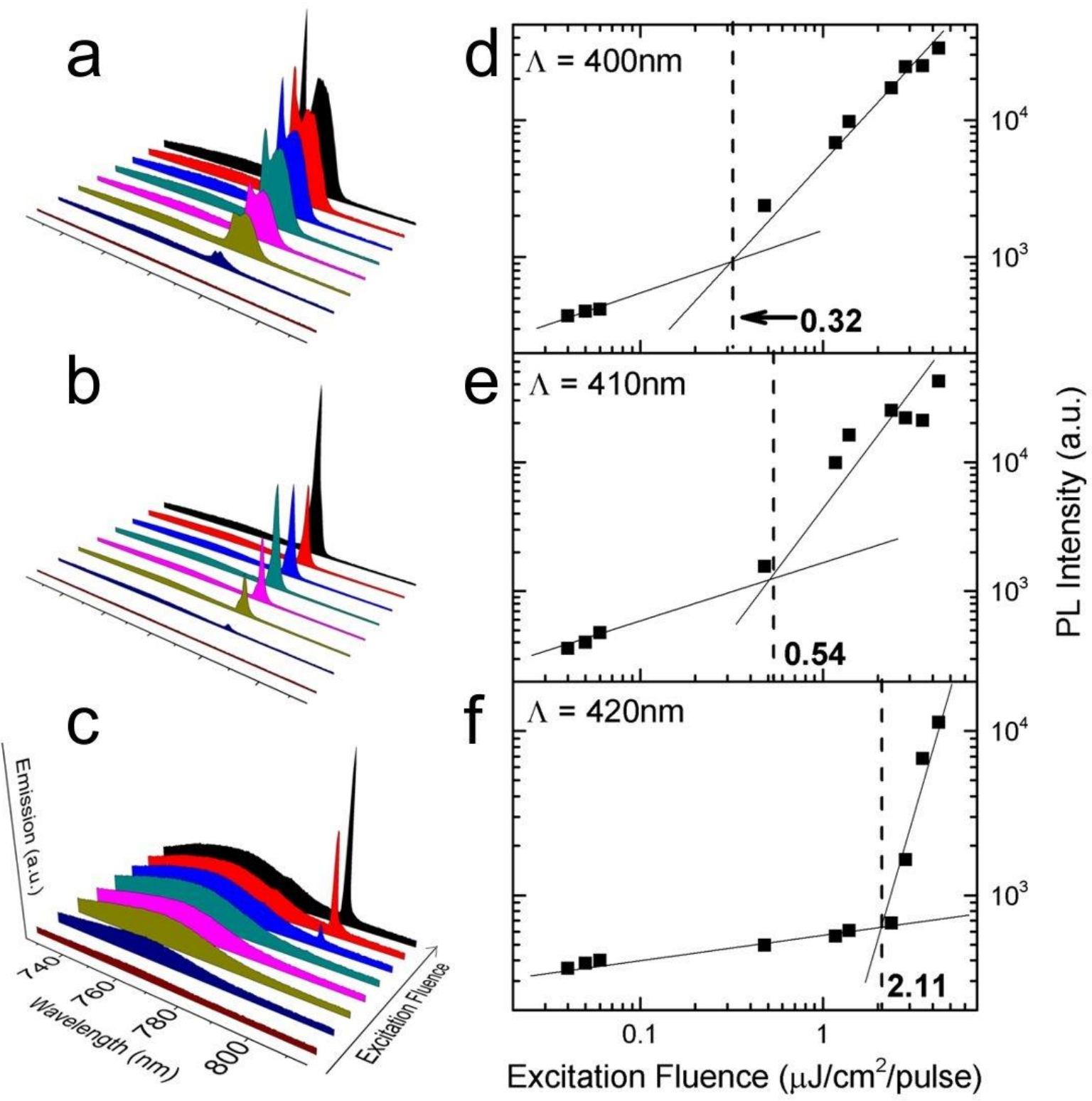

Figure 2. (a-c) Emission from a full DFB structure (glass substrate with a corrugated polymer resist and evaporated perovskite) with (a) $\Lambda=400 \mathrm{~nm}$, (b) $410 \mathrm{~nm}$, and (c) $420 \mathrm{~nm}$ periodicity 


\section{WILEY-VCH}

(all on the same perovskite film) upon optically pumping at $\lambda=532 \mathrm{~nm}$ for excitation fluences varying from 0.04 to $4.35 \mu \mathrm{J} / \mathrm{cm}^{2} /$ pulse with 1 -ns pulses at repetition rate of $1 \mathrm{kHz}$.

(d-f) Peak intensity of the amplified emission mode as a function of excitation fluence for the corresponding periodicities (a-c). The vertical dashed line corresponds to a threshold of $\sim 0.32$ $(\Lambda=400 \mathrm{~nm}), \sim 0.54(\Lambda=410 \mathrm{~nm})$, and $\sim 2.11(\Lambda=420 \mathrm{~nm}) \mu \mathrm{J} / \mathrm{cm}^{2} /$ pulse.

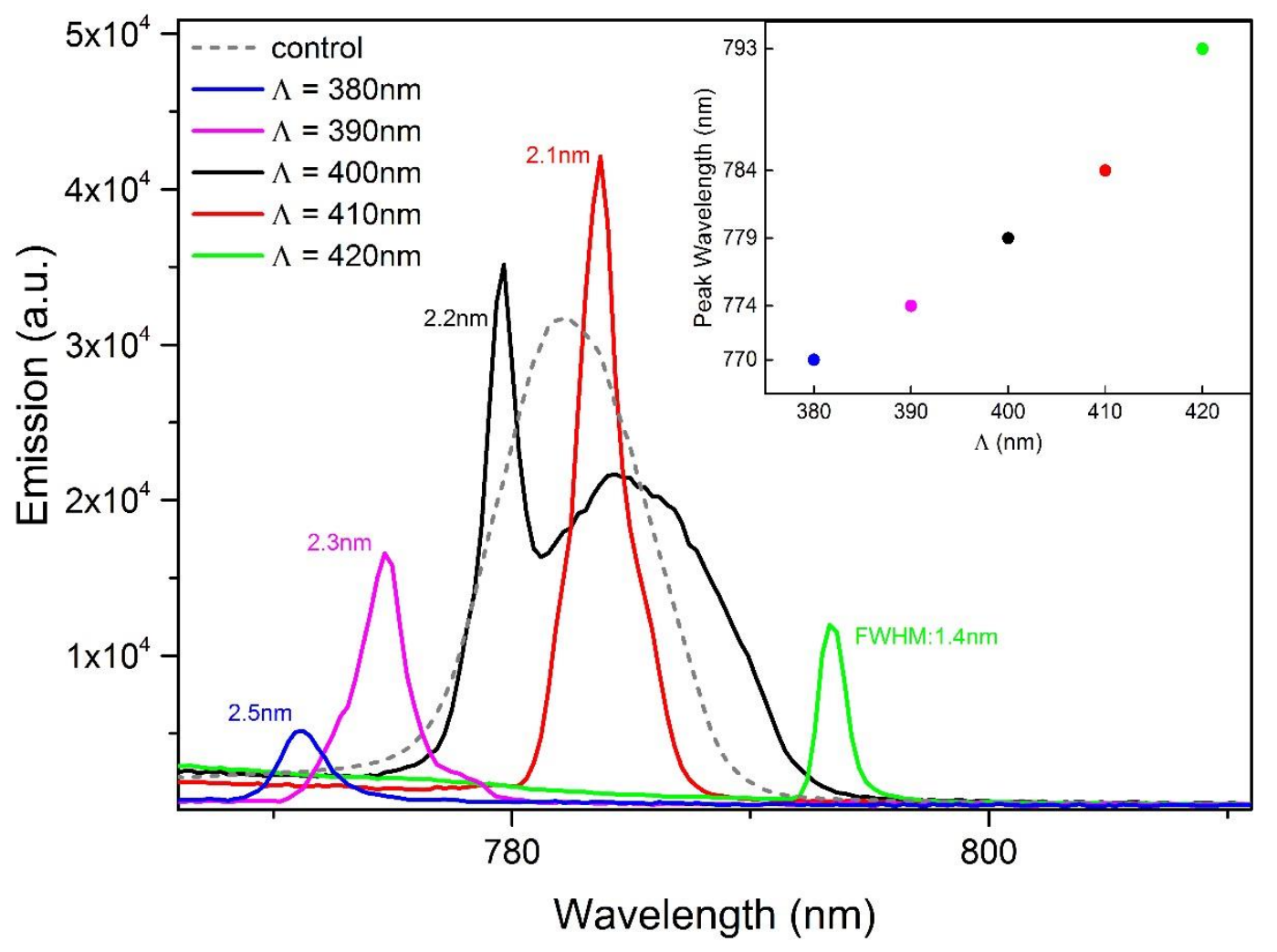

Figure 3. Magnified emission spectra for $\Lambda=400,410$, and $420 \mathrm{~nm}$ at highest fluence $\left(4.35 \mu \mathrm{J} / \mathrm{cm}^{2} /\right.$ pulse $)$. The dashed line is a control device at the same fluence from an unpatterned part of the film. 


\section{WILEY-VCH}

TOC

A general strategy for the in-plane structuring of organic-inorganic perovskite films is presented. The method is used to fabricate an industrially relevant distributed feedback (DFB) cavity, which is a critical step towards all-electrically pumped injection laser diodes. This approach opens the prospects of perovskite materials for much improved optical control in LEDs, solar cells and also toward applications as optical devices.

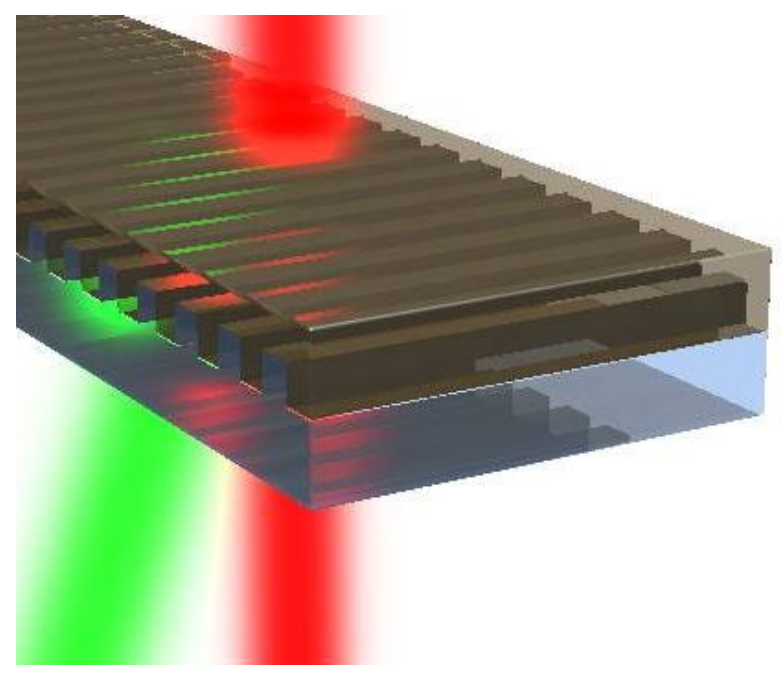




\section{WILEY-VCH}

Copyright WILEY-VCH Verlag GmbH \& Co. KGaA, 69469 Weinheim, Germany, 2013.

\section{Supporting Information}

Structured Organic-Inorganic Perovskite toward a Distributed Feedback Laser

By Michael Saliba ${ }^{1 \dagger}$, Simon M. Wood ${ }^{2}$, Jay B. Patel ${ }^{1}$, Pabitra K. Nayak ${ }^{1}$, Jian Huang ${ }^{1}$, Jack A. Alexander-Webber ${ }^{1}$, Bernard Wenger ${ }^{1}$, Samuel D. Stranks ${ }^{l}$, Maximilian T. Hörantner ${ }^{l}$, Jacob Tse-Wei Wang ${ }^{1}$, Robin J. Nicholas ${ }^{l}$, Laura M. Herz ${ }^{1}$, Michael B. Johnston ${ }^{l}$, Stephen M. Morris $^{2}$, Henry J. Snaith ${ }^{*}$, and Moritz K. Riede ${ }^{1^{*}}$

${ }^{1}$ Clarendon Laboratory, University of Oxford, Parks Road, Oxford, OX1 3PU, UK

${ }^{2}$ Department of Engineering Science, University of Oxford, Parks Road, Oxford, OX1 3PJ, UK

\section{Corresponding authors}

* henry.snaith@physics.ox.ac.uk, moritz.riede@physics.ox.ac.uk

\section{Present Address}

'Laboratory of Photonics and Interfaces, École polytechnique fédérale de Lausanne, Station 6, 1015 Lausanne, Switzerland 


\section{WILEY-VCH}
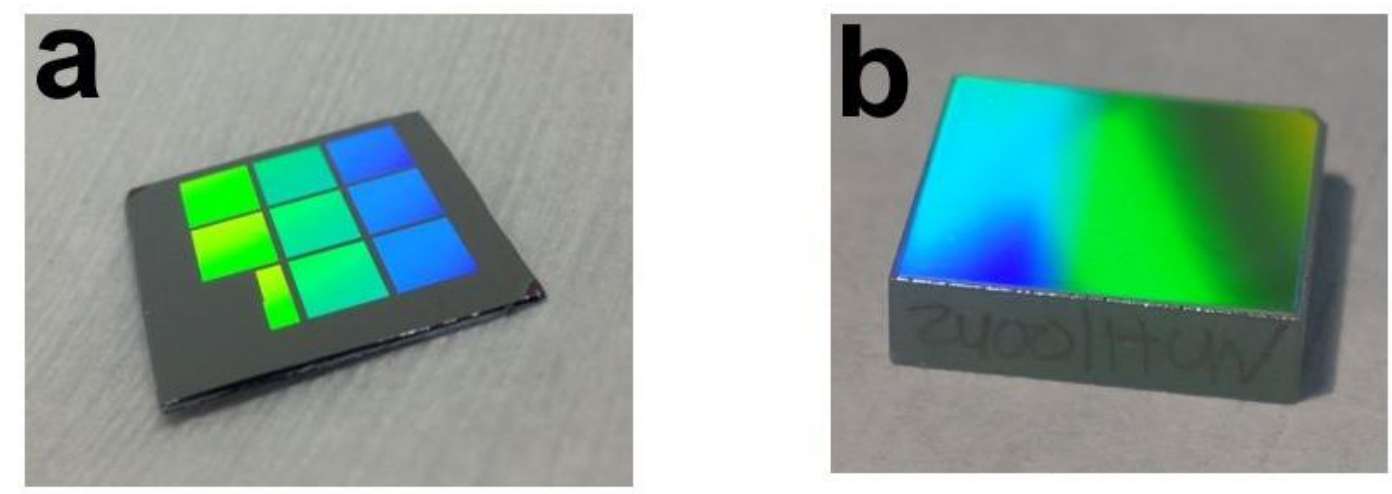

Figure S1. (a) Multistamp containing 8 different corrugation patterns with periodicities varying from 370 to $440 \mathrm{~nm}$ in steps of $10 \mathrm{~nm}$ covering an area of $0.27 \mathrm{~cm}^{2}$ per pattern. (The pattern in the bottom left was mainly left unpatterned to allow for a large area for control experiments.) (b) Thorlabs diffraction grating with a periodicity of $\sim 416 \mathrm{~nm}$ covering an area of $1.6 \mathrm{~cm}^{2}$. 


\section{WILEY-VCH}
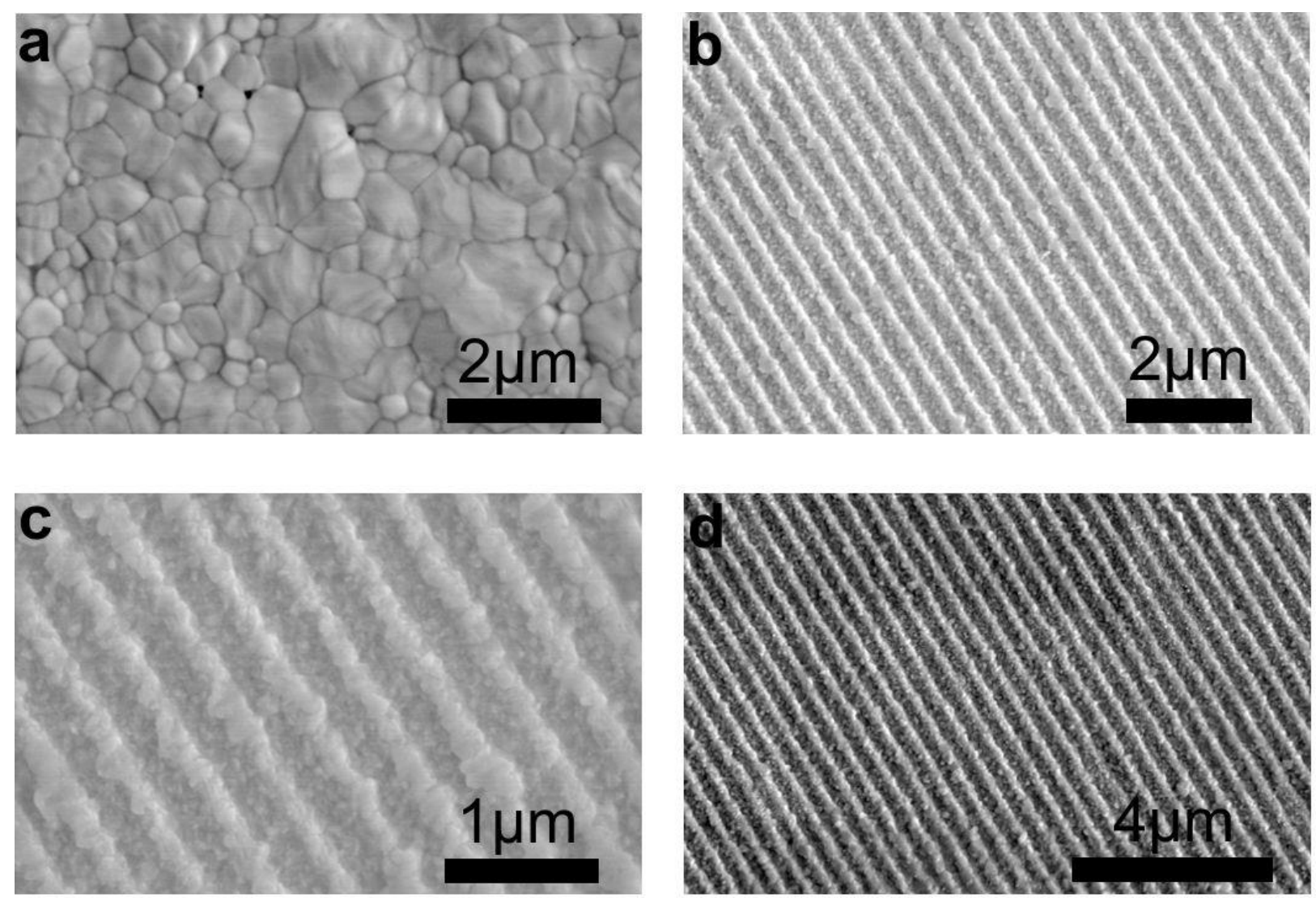

Figure S2. SEM top view of a $120 \mathrm{~nm}$ thick evaporated perovskite layers on (a) an unpatterned substrate and (b-d) a patterned substrate with $\Lambda \sim 416 \mathrm{~nm}$

For Figure S3, we used a large-area single stamp $\left(1.6 \mathrm{~cm}^{2}\right)$ with a periodicity of $\sim 416 \mathrm{~nm}$ with a slightly lower corrugation depth and evaporated accordingly a thinner $80 \mathrm{~nm}$ perovskite film. Indeed, our observations are repeated in this system where we observed a clear amplified emission peak at $796 \mathrm{~nm}$ with FWHM of $1.4 \mathrm{~nm}$, and a threshold at $\sim 1 \mu \mathrm{J} / \mathrm{cm}^{2} /$ pulse. The experiment was repeated 3 times and showed the same trend each time. 
WILEY-VCH
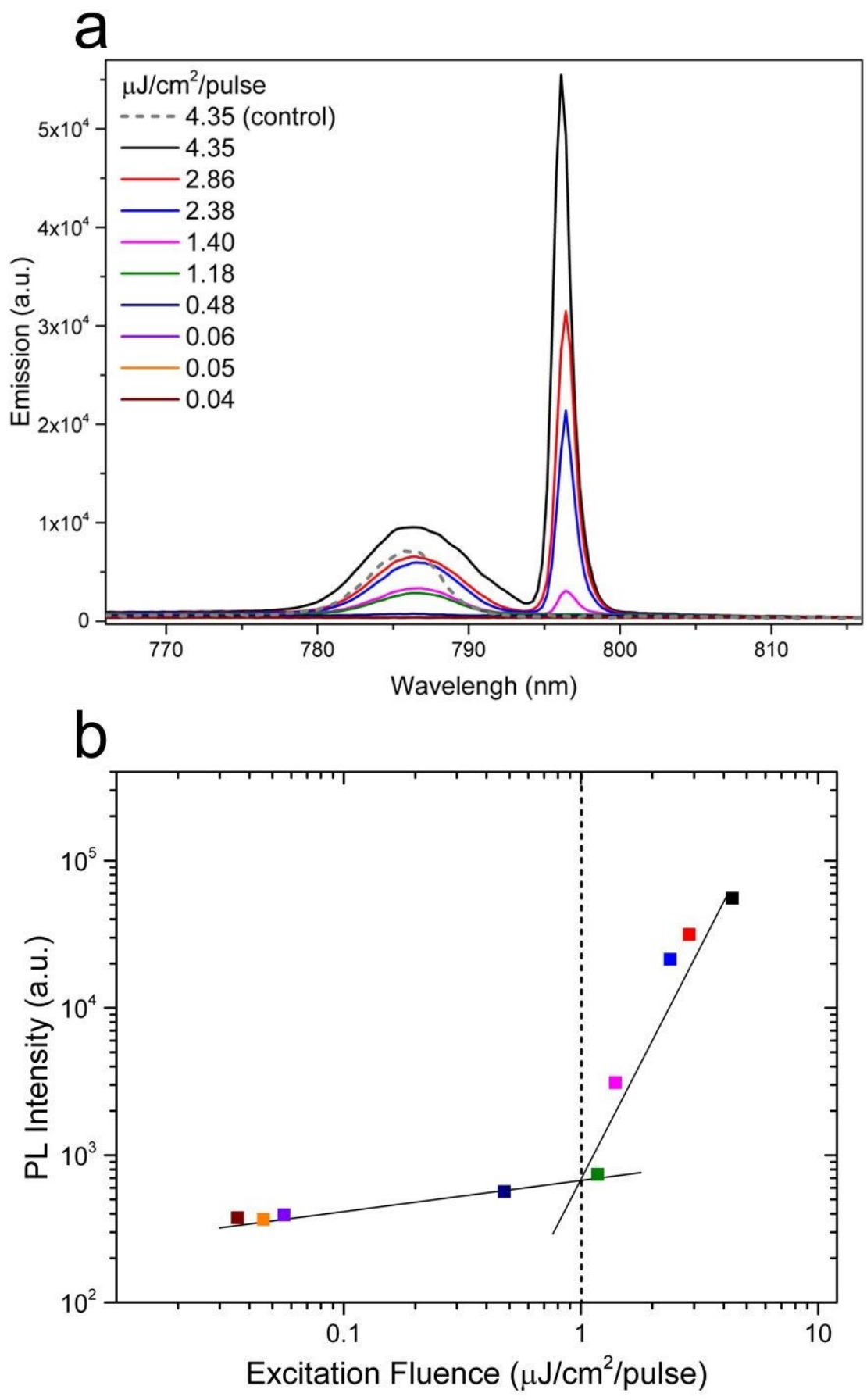

Figure S3. (a) Emission from a full DFB structure (glass substrate with a corrugated polymer resist and evaporated perovskite) with $\Lambda \sim 416 \mathrm{~nm}$ periodicity upon optically pumping at $\Lambda=532 \mathrm{~nm}$ for varying fluences with 1 -ns pulses at repetition rate of $1 \mathrm{kHz}$. The curves for fluences below $0.5 \mu \mathrm{J} / \mathrm{cm}^{2} /$ pulse overlap as there is almost no signal anymore at such low excitations.

(b) Extracted PL intensity at the amplified emission peak (796 nm with FWHM of $1.4 \mathrm{~nm}$ ) as a function of excitation fluence. The threshold is derived to be at $\sim 1 \mu \mathrm{J} / \mathrm{cm}^{2} /$ pulse. 


\section{WILEY-VCH}

Table S1. Simulated fundamental DFB modes for different periodicities $\Lambda$. The effective indices of the waveguide modes are estimated by solving numerically the phase-matching conditions for a three-layer waveguide ${ }^{[1]}$ consisting of a polymer substrate, a 100-nm thick perovskite film and air as the cover medium. The refractive indices of the individual layers are assumed as fixed at $780 \mathrm{~nm}$.

\begin{tabular}{|c|c|c|c|c|}
\hline Mode & $n_{\text {eff }}$ & $\boldsymbol{\Lambda = 4 0 0} \mathbf{n m}$ & $\boldsymbol{\Lambda = 4 1 0} \mathbf{n m}$ & $\boldsymbol{\Lambda}=\mathbf{4 2 0} \mathbf{n m}$ \\
\hline TE0 & 1.916 & 766 & 785 & 805 \\
\hline TM0 & 1.541 & 616 & 632 & 647 \\
\hline
\end{tabular}




\section{WILEY-VCH}

a

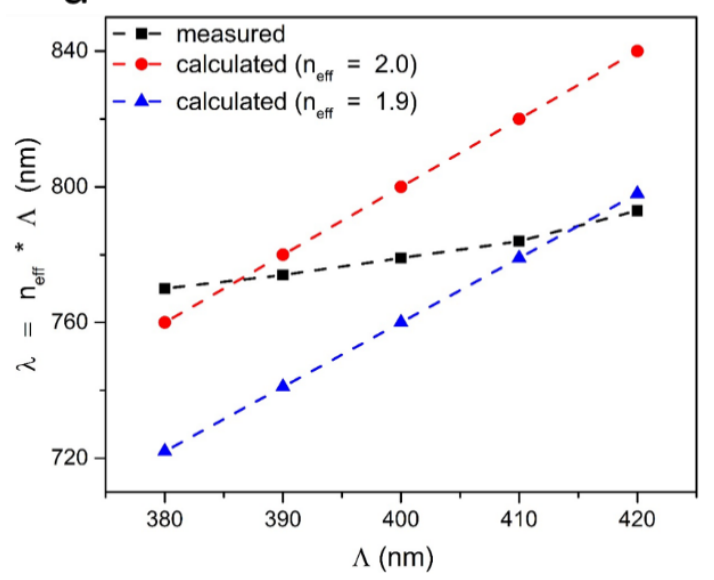

b

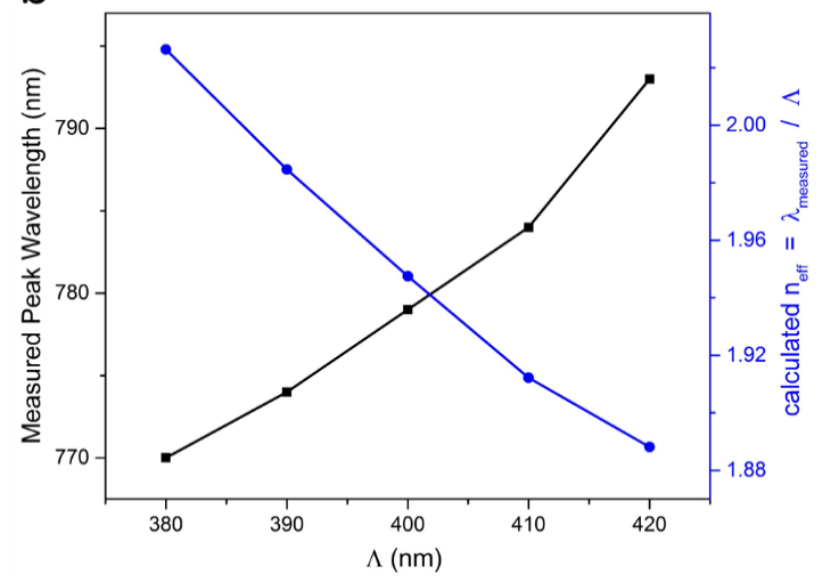

Figure S4. (a) Measured peak wavelength (squares) as a function of the periodicity $\Lambda$ Calculated modes $\lambda=n_{\text {eff }} \times \Lambda$ (second order Braff scattering) with constant $n_{\text {eff }}=1.9$ (triangles) and 2.0 (circles).

(b) Measured modes (squares) as a function of the periodicity $\Lambda$

Calculated $n_{\text {eff }}=\lambda_{\text {measured }} / \Lambda$ (circles) using the measured modes indicating a drop in the effective refractive index with increasing wavelength. 
WILEY-VCH

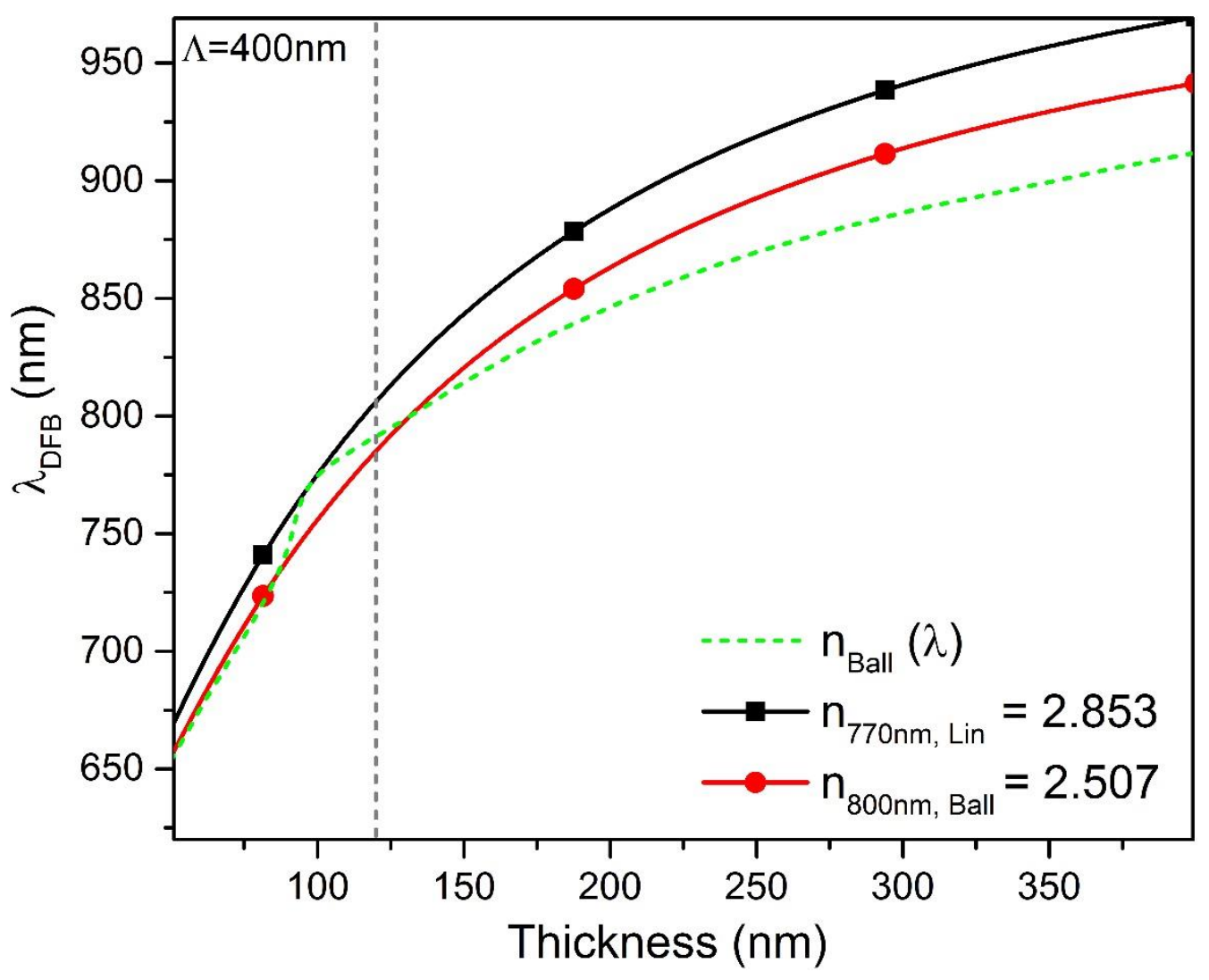

Figure S5. Estimated lasing wavelength for a DFB grating with a periodicity of $400 \mathrm{~nm}$ over the film thickness using the smallest value for the refractive indices of perovskite in the 770 $800 \mathrm{~nm}$ range with $\mathrm{n}_{800 \mathrm{~nm}, \text { Ball }}=2.507$ (squares) and the largest value $\mathrm{n}_{770 \mathrm{~nm}, \mathrm{Lin}}=2.853$ (circles) according to Ball et al. ${ }^{[2]}$ and Lin et al. ${ }^{[3]}$ The dashed curve takes the dispersion relation into account as measured by Ball et al. ${ }^{[2]}$ The vertical dashed line indicates the experimental film thickness emphasizing the importance of accurate optical data.

[1] K. Okamoto, Fundamentals of optical waveguides, Academic press, London, UK, 2006.

[2] J. M. Ball, S. D. Stranks, M. T. Hoerantner, S. Huettner, W. Zhang, E. J. W. Crossland, I. Ramirez, M. Riede, M. B. Johnston, R. H. Friend, H. J. Snaith, Energy \& Environmental Science 2015, 8, 602.

[3] Q. Q. Lin, A. Armin, R. C. R. Nagiri, P. L. Burn, P. Meredith, Nat Photonics 2015, 9, 106. 